\title{
Erratum to: Effects of pruning on knotty core taper and form of Pinus radiata and Pinus pinaster
}

\author{
Andrea Hevia $^{1}$ - Juan Gabriel Álvarez-González ${ }^{2} \cdot$ Juan Majada $^{1}$
}

Published online: 7 April 2016

(c) Springer-Verlag Berlin Heidelberg 2016

\section{Erratum to: Eur. J. Wood Prod. \\ DOI 10.1007/s00107-016-1019-0}

Due to an error during conversion of Geographic Units (Latitude, Longitude) from UTM coordinates, information given for location of the experimental plots in Table 1 of the manuscript is not correct.

The data of location of the plots should be replaced by:

\begin{tabular}{ll}
\hline Experimental plot & Location \\
\hline Pinus radiata & \\
La Campa & $43^{\circ} 24^{\prime} \mathrm{N}, 5^{\circ} 28^{\prime} \mathrm{W}$ \\
Cabada & $43^{\circ} 25^{\prime} \mathrm{N}, 6^{\circ} 33^{\prime} \mathrm{W}$ \\
Santa Catalina & $43^{\circ} 31^{\prime} \mathrm{N}, 6^{\circ} 6^{\prime} \mathrm{W}$ \\
Fabal & $43^{\circ} 12^{\prime} \mathrm{N}, 6^{\circ} 53^{\prime} \mathrm{W}$ \\
Pinus pinaster & \\
Valsera & $43^{\circ} 33^{\prime} \mathrm{N}, 6^{\circ} 12^{\prime} \mathrm{W}$ \\
Barcia & $43^{\circ} 31^{\prime} \mathrm{N}, 6^{\circ} 28^{\prime} \mathrm{W}$ \\
Monteagudo & $43^{\circ} 31^{\prime} \mathrm{N}, 6^{\circ} 6^{\prime} \mathrm{W}$ \\
Uria & $43^{\circ} 4^{\prime} \mathrm{N}, 6^{\circ} 51^{\prime} \mathrm{W}$ \\
\hline
\end{tabular}

The online version of the original article can be found under doi:10.1007/s00107-016-1019-0.

Andrea Hevia

ahevia@cetemas.es

1 Sustainable Forest Management Area, Forest and Wood

Technology Research Centre (CETEMAS), Finca

Experimental "La Mata” s/n, 33820 Grado, Spain

2 Sustainable Forest Management Unit, Agroforestry

Engineering Department, High Polytechnic School,

University of Santiago de Compostela, Campus Universitario

s/n, 27002 Lugo, Spain
Site Index has been recalculated at the time of establishment of the pruning plots. Both changes are not substantial for the study described and do not affect the results and final conclusions presented in this work.

On the next page, the whole Table is presented. 
Table 1 Details of the network of plots and data used in this study

\begin{tabular}{|c|c|c|c|c|c|c|c|c|c|c|c|c|}
\hline \multirow{3}{*}{$\begin{array}{l}\text { Experimental } \\
\text { plot }\end{array}$} & \multirow[t]{3}{*}{ Location } & \multirow{3}{*}{$\begin{array}{l}\text { Site Index* } \\
\text { (m) }\end{array}$} & \multicolumn{2}{|c|}{ At time of installation } & \multicolumn{8}{|c|}{ At time of inventory } \\
\hline & & & \multirow{2}{*}{$\begin{array}{l}\text { Age } \\
\text { (years) }\end{array}$} & \multirow{2}{*}{$\begin{array}{l}\text { Stocking } \\
\left(\text { trees } \mathrm{ha}^{-1}\right)\end{array}$} & \multirow{2}{*}{$\frac{H^{*}(\mathrm{~m})}{\text { Mean }}$} & \multirow{2}{*}{$\frac{D^{*}(\mathrm{~cm})}{\text { Mean }}$} & \multicolumn{3}{|c|}{$d o s_{\mathrm{j}}^{*}(\mathrm{~cm})$} & \multicolumn{3}{|c|}{$h_{D O S_{\mathrm{j}}}{ }^{*}(\mathrm{~m})$} \\
\hline & & & & & & & Mean & Max & Min & Mean & $\operatorname{Max}$ & Min \\
\hline \multicolumn{13}{|l|}{ Pinus radiata } \\
\hline La Campa & $43^{\circ} 24^{\prime} \mathrm{N}, 5^{\circ} 28^{\prime} \mathrm{W}$ & 22.2 & 9 & 1187 & 11.9 & 16.8 & 18.0 & 28.0 & 9.0 & 1.7 & 5.7 & 0.3 \\
\hline Cabada & $43^{\circ} 25^{\prime} \mathrm{N}, 6^{\circ} 33^{\prime} \mathrm{W}$ & 26.1 & 7 & 1261 & 11.0 & 14.4 & 16.5 & 27.2 & 8.7 & 1.4 & 4.1 & 0.3 \\
\hline Santa Catalina & $43^{\circ} 31^{\prime} \mathrm{N}, 6^{\circ} 6^{\prime} \mathrm{W}$ & 21.3 & 8 & 1440 & 10.8 & 15.4 & 16.7 & 26.9 & 9 & 1.2 & 3.6 & 0.3 \\
\hline Fabal & $43^{\circ} 12^{\prime} \mathrm{N}, 6^{\circ} 53^{\prime} \mathrm{W}$ & 23.6 & 10 & 1356 & 11.4 & 14.8 & 16.9 & 28.3 & 8.1 & 1.3 & 4.0 & 0.3 \\
\hline \multicolumn{13}{|l|}{ Pinus pinaster } \\
\hline Valsera & $43^{\circ} 33^{\prime} \mathrm{N}, 6^{\circ} 12^{\prime} \mathrm{W}$ & 17.9 & 8 & 1352 & 9.99 & 14.8 & 16.4 & 24 & 8.9 & 1.3 & 3.7 & 0.2 \\
\hline Barcia & $43^{\circ} 31^{\prime} \mathrm{N}, 6^{\circ} 28^{\prime} \mathrm{W}$ & 13.5 & 11 & 1379 & 7.9 & 13.2 & 15.9 & 22.5 & 9.0 & 1.3 & 2.8 & 0.3 \\
\hline Monteagudo & $43^{\circ} 31^{\prime} \mathrm{N}, 6^{\circ} 6^{\prime} \mathrm{W}$ & 13.2 & 8 & 1670 & 6.7 & 12.9 & 15.0 & 22.5 & 10.1 & 0.96 & 2.3 & 0.3 \\
\hline Uria & $43^{\circ} 4^{\prime} \mathrm{N}, 6^{\circ} 51^{\prime} \mathrm{W}$ & 15.9 & 7 & 1802 & 6.5 & 9.7 & 13.2 & 14.5 & 10.6 & 0.8 & 0.9 & 0.6 \\
\hline
\end{tabular}

* $H$ total tree height, $\mathrm{m} ; D$ diameter outside bark at breast height $\left(1.30 \mathrm{~m}\right.$ above ground), $\mathrm{cm} ; d_{0} s_{\mathrm{j}}$ diameter over stubs at height of pruned whorl $\mathrm{j}$, $\mathrm{cm} ; h_{D O S \mathrm{j}}$ height above ground at specific point $j$ on the stem, $\mathrm{m}$. Site Index estimated using the equations defined by Canga (2008) and ÁlvarezÁlvarez et al. (2011) for $P$. radiata and $P$. pinaster, respectively 